\title{
Postprandial glucose and insulin profiles following a glucose-loaded meal in cats and dogs
}

\author{
Adrian K. Hewson-Hughes*, Matthew S. Gilham, Sarah Upton, Alison Colyer, Richard Butterwick \\ and Andrew T. Miller \\ Waltham Centre for Pet Nutrition, Waltham-on-the-Wolds, Melton Mowbray, Leicestershire LE14 4RT, UK
}

(Received 15 October 2010 - Revised 11 January 2011 - Accepted 28 January 2011)

\section{Abstract}

Data from intravenous (i.v.) glucose tolerance tests suggest that glucose clearance from the blood is slower in cats than in dogs. Since different physiological pathways are activated following oral administration compared with i.v. administration, we investigated the profiles of plasma glucose and insulin in cats and dogs following ingestion of a test meal with or without glucose. Adult male and female cats and dogs were fed either a high-protein (HP) test meal ( $15 \mathrm{~g} / \mathrm{kg}$ body weight; ten cats and eleven dogs) or a HP + glucose test meal (13 g/kg body-weight HP diet $+2 \mathrm{~g} / \mathrm{kg}$ body-weight D-glucose; seven cats and thirteen dogs) following a $24 \mathrm{~h}$ fast. Marked differences in plasma glucose and insulin profiles were observed in cats and dogs following ingestion of the glucose-loaded meal. In cats, mean plasma glucose concentration reached a peak at $120 \mathrm{~min}(10 \cdot 2,95 \%$ CI $9 \cdot 7,10 \cdot 8 \mathrm{mmol} / \mathrm{l})$ and returned to baseline by 240 min, but no statistically significant change in plasma insulin concentration was observed. In dogs, mean plasma glucose concentration reached a peak at 60 min $(6 \cdot 3,95 \% \mathrm{CI}$ $5.9,6.7 \mathrm{mmol} / \mathrm{l}$ ) and returned to baseline by $90 \mathrm{~min}$, while plasma insulin concentration was significantly higher than pre-meal values from 30 to 120 min following the glucose-loaded meal. These results indicate that cats are not as efficient as dogs at rapidly decreasing high blood glucose levels and are consistent with a known metabolic adaptation of cats, namely a lack of glucokinase, which is important for both insulin secretion and glucose uptake from the blood.

\section{Key words: Feline: Canine: Glycaemia: Insulinaemia}

Inspection of data derived from intravenous glucose tolerance tests suggests that glucose clearance from the blood is slower in cats than in dogs. Thus, following an intravenous glucose bolus ( $1 \mathrm{~g} / \mathrm{kg}$ body weight), plasma glucose levels took up to $90 \mathrm{~min}$ to return to baseline in cats, whereas a return to baseline was observed after $40-60 \mathrm{~min}$ in $\operatorname{dogs}{ }^{(1,2)}$. While intravenous administration of glucose is widely used as a means of assessing glucose tolerance, we were interested in measuring the changes in blood glucose and insulin profiles following food intake since ingestion is considered to give a more physiologically complete picture than intravenous administration, as it also accounts for effects of intestinal absorption and activation of the enteroinsular axis (i.e. the neural and hormonal responses that influence insulin secretion) on the resulting glucose and insulin plasma profiles. A number of studies have measured postprandial plasma glucose and insulin profiles in cats and dogs, typically in response to meals of dry food containing different types of $\operatorname{starch}^{(3-5)}$. In contrast to the rapid changes in blood glucose and insulin concentrations observed following intravenous glucose administration, these meal response studies have shown smaller changes in glucose and insulin concentrations, which occur gradually over a longer period of time (e.g. glucose and insulin levels peaking several hours after the meal). In order to evaluate dynamic changes in blood glucose and insulin concentrations in response to a physiologically informative route of administration, we have determined postprandial plasma glucose and insulin concentrations in response to a meal with or without glucose.

\section{Methods}

\section{Animals}

Initially, twenty-four healthy adult domestic shorthair cats and twenty-five healthy adult dogs (six Labrador retrievers (LR) and nineteen Miniature Schnauzers (MS)) were selected for the present study, but samples were not obtained from six cats and one dog; details of animals allocated to each diet are given below. Cats were housed individually

Abbreviations: FN, female neutered; GK, glucokinase; HP, high protein; HP + G, high protein + glucose; LR, Labrador retrievers; MN, male neutered; MS, Miniature Schnauzers.

*Corresponding author: Dr A. K. Hewson-Hughes, fax +44 1664 415440, email adrian.hewson-hughes@effem.com 
in environmentally enriched lodges and group-exercised/ socialised for several hours each day, except for the test meal day. Before the test meal day, dogs were housed in pairs, allowed several hours of paddock exercise and walked on-lead each day; on the test meal day, dogs were individually housed and given calm social interaction but no exercise. All animals underwent habituation programmes to accustom them to the blood sampling procedures to be carried out on the test meal day. The experimental procedures were approved by the Waltham Ethical Review Committee and performed in accordance with UK Home Office legislation.

\section{Diet}

The test diet was a canned diet made to a standard loaf-based recipe, enriched with chicken breast and soya isolate to increase the protein content, manufactured at Mars Petcare, Verden, Germany. The macronutrient composition of the diet was as follows ( $\mathrm{g} / 100 \mathrm{~g}$ as is): moisture, $81 \cdot 4$; protein, $12 \cdot 7$; fat $2 \cdot 5$; $\mathrm{N}$ free extract (carbohydrate) 1.4 . For the test meal, this diet was either fed without modification (high protein (HP), HP test meal) or with the addition of D-glucose (HP + G test meal; Sigma Chemical Company, Dorset, UK). Specifically, one group of cats and one group of dogs were offered a test meal of $15 \mathrm{~g} \mathrm{HP}$ diet $/ \mathrm{kg}$ body weight, while the other group of cats and dogs were offered a test meal of $13 \mathrm{~g} \mathrm{HP}$ diet/kg body weight $+2 \mathrm{~g} / \mathrm{kg}$ body-weight D-glucose.

\section{Experimental procedures}

Cats and dogs were assigned to groups and received either the HP test meal (three female neutered (FN) and seven male neutered $(\mathrm{MN})$ cats; two FN LR, one MN LR, three FN MS and five MN MS dogs) or the HP $+\mathrm{G}$ test meal (two FN and five MN cats; two FN LR, one MN LR, four FN MS, one FN MS and five MN MS dogs). Following an overnight (24h) fast, animals were weighed (to calculate how much test meal to offer), and a cannula was inserted into a cephalic vein. Blood samples were taken, via the cannula, 90 (dog only), 60 and $30 \mathrm{~min}$ before, and 30, 60, 90, 120, 150, 180, 210, 240, 270, 300 and 360 min after the test meal was offered. The test meal was offered at $t=0$ and was consumed within 10 min. Cannula patency was maintained by flushing with $0.5 \mathrm{ml}$ of heparinised saline (heparin $100 \mathrm{IU} / \mathrm{ml}$ in $0.9 \%$ $\mathrm{NaCl}$ ) after each sample. Before collecting each sample, $0.3 \mathrm{ml}$ of blood were withdrawn and discarded to avoid any dilution of the analytes of interest by saline in the cannula. Blood $(1.3 \mathrm{ml})$ was collected into EDTA and sodium fluoride-potassium oxalate tubes and kept on ice until centrifugation (within $30 \mathrm{~min}$ of collection) at $2000 \mathrm{~g}$ for $10 \mathrm{~min}$ at $4^{\circ} \mathrm{C}$. Plasma from EDTA tubes was stored at $-80^{\circ} \mathrm{C}$ until insulin concentration was determined by RIA (porcine insulin kit, PI-12K (Millipore (UK) Limited, Watford, Hertfordshire, UK) for cat samples; human insulin kit, DSL1600 (Diagnostic System Laboratories, Inc., Webster, TX, USA; Beckman Coulter (UK) Limited, High Wycombe, Buckinghamshire) for dog samples). Plasma from sodium fluoride-potassium oxalate tubes was assayed immediately for glucose using an automated analyser (AU400; Olympus Instruments, Beckman Coulter (UK) Limited High Wycombe, Bucks, UK).

\section{Statistical analysis}

Plasma glucose and insulin profiles were analysed using mixed model analysis to take into account the repeated measures on each animal. Fixed effects of species, diet, time from meal and their interactions were explored. Post boc analysis was performed using Tukey's honestly significant difference procedure for unequal repetitions between means to maintain the overall significance level at 5\% and account for multiple comparisons. Statistical analysis was carried out using Statistica software, version 8 (Statsoft Limited, Bedford, UK). Graphs of response profiles are presented with means and $95 \% \mathrm{CI}$.

\section{Results}

Plasma glucose and insulin concentrations measured before the test meal (i.e. after a $24 \mathrm{~h}$ fast) were not significantly different in cats and dogs. Following ingestion of the HP meal, no statistically significant changes in plasma glucose or insulin concentrations were observed compared with the pre-meal concentrations in either cats or dogs, and the concentrations of both glucose and insulin were very similar in both species (Figs. 1 and 2). In contrast, marked changes in both plasma glucose and insulin concentrations were observed following ingestion of the $\mathrm{HP}+\mathrm{G}$ meal, and the profiles were very different between cats and dogs. In both species, plasma glucose concentration was significantly higher than pre-meal values by $60 \mathrm{~min}$ after the meal (cats, $P=0.001$; dogs, $P=0.00058)$ and was significantly higher in cats than in dogs $(P=0.02)$. In dogs, plasma glucose concentration began to decline after this time and was not significantly greater than pre-meal values from 90 min onwards, while in cats, plasma glucose concentration continued to rise before peaking at $120 \mathrm{~min}$ (Fig. 1). Plasma glucose concentration in cats was not statistically different from pre-meal concentration from 240 min onwards (although an increase in mean concentration at $270 \mathrm{~min}$ is shown in Fig. 1, it was not statistically significant). In dogs, plasma insulin concentration was significantly elevated above pre-meal values from 30 to $120 \mathrm{~min}$ following the $\mathrm{HP}+\mathrm{G}$ meal ( $P \leq 0 \cdot 001$; Fig. 2). In contrast and despite the large increases in plasma glucose concentration observed in cats, mean plasma insulin concentration in this species rose gradually following the $\mathrm{HP}+\mathrm{G}$ meal, with a peak at $210 \mathrm{~min}$ but was never statistically different from pre-meal values (Fig. 2).

\section{Discussion}

The most striking observation from the present study is the very different glucose and insulin profiles observed between cats and dogs following ingestion of a glucose-loaded meal. For a very large increase in plasma glucose concentration, the insulin response appears 'blunted' in cats, while a rapid and large insulin response was observed in dogs in response to a relatively small increase in plasma glucose concentration. 




Fig. 1. Plasma glucose concentration of cats $(O, \bullet)$ and dogs $(\triangle, \boldsymbol{\Delta})$ before and after a high-protein $(\mathrm{HP}) \mathrm{meal}(15 \mathrm{~g} / \mathrm{kg}$ body weight; $\bigcirc, \triangle)$ or the same $\mathrm{HP}$ diet ( $13 \mathrm{~g} / \mathrm{kg}$ body weight) to which D-glucose $(2 \mathrm{~g} / \mathrm{kg}$ body weight) was added just before feeding $(\mathrm{HP}+\mathrm{G} ; \boldsymbol{\bullet}, \boldsymbol{\Delta})$. Values are means, with $95 \% \mathrm{Cl}$ represented by vertical bars. *Mean values were significantly different from baseline ( -60 and $-30 \mathrm{~min} ; P \leq 0.001)$. †Mean values were significantly different from baseline ( $-60 \mathrm{~min} ; P=0.000058)$. $¥$ Mean values were significantly different from dogs fed the HP $+\mathrm{G}$ meal $(P \leq 0.02)$.

These differences are consistent with known metabolic adaptations of cats to evolution on a low-carbohydrate diet.

The longer time for plasma glucose concentration to peak and the higher peak concentration observed in cats compared with dogs possibly reflect a combination of slower glucose absorption from the intestine to the circulation and subsequently slower cellular uptake of glucose from the blood. The rate of glucose uptake is higher in the intestine of suckling kittens compared with kittens weaned onto solid food $^{(6)}$. This age-related decline in capacity for glucose uptake in cats could not be prevented by feeding a highcarbohydrate diet $(57,93 \%$ of which was from added glucose) to young cats ${ }^{(7)}$, which is in contrast to omnivorous species that up-regulate glucose transport activities in response to a high-carbohydrate $\operatorname{diet}^{(8,9)}$. The effect of digestible carbohydrate on glucose uptake by the canine intestine has not been studied, but dogs fed a diet containing fermentable fibre (beet pulp and oligofructose) had a 95\% higher capacity for carrier-mediated glucose uptake than dogs fed a non-fermentable fibre (cellulose), indicating the potential for nutritional up-regulation of glucose uptake by the small intestine in dogs ${ }^{(10)}$

Following absorption from the gastrointestinal tract, removal of glucose from the blood by active transport into cells is maintained by a concentration gradient, as intracellular glucose is rapidly converted to glucose 6-phosphate by

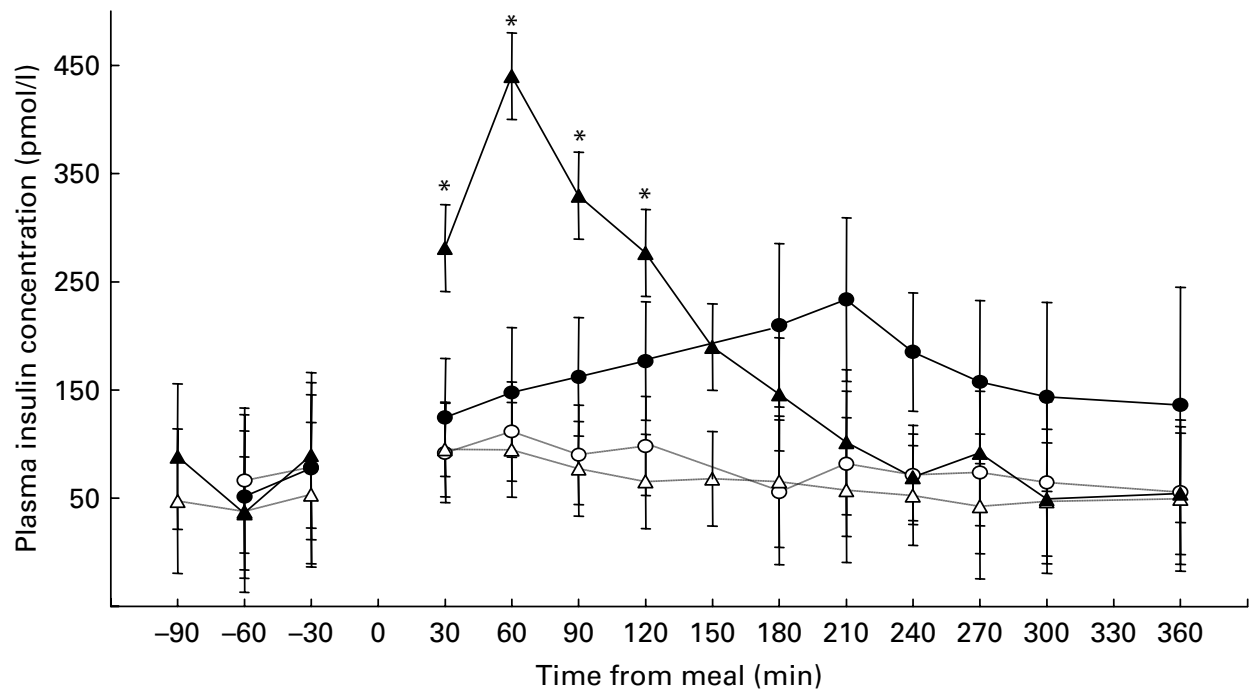

Fig. 2. Plasma insulin concentration of cats $(\bigcirc, \bullet)$ and dogs $(\Delta, \boldsymbol{\Delta})$ before and after a high-protein $(\mathrm{HP}) \mathrm{meal}(15 \mathrm{~g} / \mathrm{kg}$ body weight; $\bigcirc, \Delta)$ or the same $\mathrm{HP}$ diet ( $13 \mathrm{~g} / \mathrm{kg}$ body weight) to which D-glucose $(2 \mathrm{~g} / \mathrm{kg}$ body weight) was added just before feeding $(\bullet, \mathbf{\Delta})$. Values are means, with $95 \% \mathrm{Cl}$ represented by vertical bars. * Mean values were significantly different from baseline $(-90,-60$ and $-30 \mathrm{~min} ; P \leq 0.001)$. 
hexokinase and/or glucokinase (GK). Hexokinase has a high affinity for glucose but low capacity, whereas GK has a lower affinity for glucose but high capacity, making it ideally suited for dealing with high glucose concentrations. However, in contrast to dogs, feline tissues appear to lack GK enzyme activity or mRNA expression $^{(11,12)}$ and may help explain the large and prolonged increase in plasma glucose concentration observed in cats following the glucose-loaded meal. Low GK activity has been reported in other carnivorous species such as barn owls and rainbow trout and is believed to contribute to the prolonged glucose elevation observed following a glucose challenge in these species ${ }^{(13,14)}$.

That the post-prandial increase in insulin concentration in cats following the glucose-loaded meal did not reach statistical significance is surprising and may be explained by individual variability in the magnitude and timing of the insulin response (e.g. at $30 \mathrm{~min}$ following the glucose-loaded meal, insulin concentration was the same as the pre-meal concentration in one cat, while in all the others it had increased markedly). While the anti-porcine antibody in the insulin assay kit used to measure feline plasma insulin concentration is $100 \%$ cross-reactive to human insulin, the differences in amino acid sequence between feline and human insulin may have resulted in an underestimation of plasma insulin concentration in cats in the present study. GK also plays an important role in the pancreatic $\beta$-cell as a 'glucose sensor' linking the detection of elevated blood glucose levels to insulin secretion through $\beta$-cell metabolism of glucose ${ }^{(15,16)}$. While GK mRNA expression has been demonstrated in feline pancreas ${ }^{(12,17)}$, it is possible that low pancreatic GK activity may explain the 'blunted' insulin response observed in cats following the glucose-loaded meal.

A further possible explanation for the limited insulin response in cats could be related to incretin hormone release (glucagon-like peptide-1) and glucose-dependent insulinotrophic polypeptide that potentiate insulin secretion ${ }^{(18)}$. In human subjects, a reduction in postprandial concentrations of glucagon-like peptide-1 appears to contribute to the impaired insulin secretion associated with type 2 diabetes ${ }^{(19)}$. The response of these hormones to food intake has not been characterised in cats or dogs and is an important missing piece of the jigsaw of the physiological response to food intake in these species, particularly in light of the differences in the insulin response to the glucose-loaded meal observed in the present study.

\section{Acknowledgements}

The authors have no conflicts of interest to declare. The present study was funded by Mars Petcare, Verden, Germany. A. K. H.-H. and A. T. M. conceived and designed the study. A. K. H.-H., M. S. G., S. U. and A. T. M. carried out the study, and A. C. carried out the statistical analysis. A. K. H.-H. wrote the manuscript with critical input and guidance from R. B. The authors would like to thank many people at the Waltham Centre for Pet Nutrition for their skills and expertise in the training, welfare and sampling of the cats and dogs in these studies.

\section{References}

1. Church DB (1980) A comparison of intravenous and oral glucose tolerance tests in the dog. Res Vet Sci 29, 353-359.

2. Hoenig M, Alexander S, Holson J, et al. (2002) Influence of glucose dosage on interpretation of intravenous glucose tolerance tests in lean and obese cats. J Vet Int Med 16, 529-532.

3. Bouchard GF \& Sunvold GD (2000) Effect of dietary carbohydrate source on postprandial plasma glucose and insulin concentration in cats. In Recent Advances in Canine and Feline Nutrition, vol. III, pp. 91-101 [GA Reinhart and DP Carey, editors]. Iams Nutrition Symposium Proceedings Wilmington, $\mathrm{OH}$ : Orange Frazer Press.

4. Appleton DJ, Rand JS, Priest J, et al. (2004) Dietary carbohydrate source affects glucose concentrations, insulin secretion and food intake in overweight cats. Nutr Res 24, 447-467.

5. de-Oliveira LD, Carciofi AC, Oliveira MCC, et al. (2008) Effects of six carbohydrate sources on diet digestibility and postprandial glucose and insulin responses in cats. J Anim Sci 86, 2237-2246.

6. Buddington RK \& Diamond J (1992) Ontogenic development of nutrient transporters in cat intestine. Am J Physiol Gastrointest Liver Physiol 263, G605-G616.

7. Buddington RK, Chen JW \& Diamond JM (1991) Dietary regulation of intestinal brush-border sugar and amino acid transport in carnivores. Am J Physiol 261, R793-R801.

8. Diamond JM \& Karasov WH (1984) Effect of dietary carbohydrate on monosaccharide uptake by mouse intestine in vitro. J Physiol 349, 419-440.

9. Scharrer E, Wolfram W, Raab W, et al. (1982) Adaptive changes of amino acid and sugar transport across the brush border of rat jejunum. In Mechanisms of Intestinal Adaptation, pp. 123-137 [JWL Robinson, RH Dowling and and E-O Riecken, editors]. Lancaster: MTP Press.

10. Buddington RK, Buddington KK \& Sunvold GD (1999) Influence of fermentable fibre on small intestinal dimensions and transport of glucose and proline in dogs. Am J Vet Res 60, 354-358.

11. Ballard FJ (1965) Glucose utilization in mammalian liver. Comp Biochem Physiol 14, 437-443.

12. Tanaka A, Inoue A, Takeguchi A, et al. (2005) Comparison of expression of glucokinase gene and activities of enzymes related to glucose metabolism in livers between cats and dogs. Vet Res Commun 29, 477-485.

13. Myers MR \& Klasing KC (1999) Low glucokinase activity and high rates of gluconeogenesis contribute to hyperglycemia in barn owls (Tyto alba) after a glucose challenge. J Nutr 129, 1896-1904.

14. Palmer TN \& Ryman BE (1972) Studies on oral glucose tolerance in fish. J Fish Biol 4, 311-319.

15. Matschinsky FM (1990) Glucokinase as glucose sensor and metabolic signal generator in pancreatic $\beta$-cells and hepatocytes. Diabetes 39, 647-652.

16. Matschinsky FM (1996) A lesson in metabolic regulation inspired by the glucokinase sensor paradigm. Diabetes $\mathbf{4 5}$, $223-241$.

17. Hiskett E, Donley V \& Schermerhorn T (2006) Glucokinase and hexokinase expression in the feline pancreas. Comp Cont Edu Pract Vet 28, 47.

18. Drucker DJ (2006) The biology of incretin hormones. Cell Metab 3, 153-165.

19. Toft-Nielsen M-B, Damholt MB, Madsbad S, et al. (2001) Determinants of the impaired secretion of glucagon-like peptide- 1 in type 2 diabetic patients. J Clin Invest 86, 3717-3723. 